ISSN 2076-393X

www.mdpi.com/journal/vaccines

Article

\title{
CD137 Enhancement of HPV Positive Head and Neck Squamous Cell Carcinoma Tumor Clearance
}

\author{
Christopher T. Lucido ${ }^{1, \dagger}$, Paola D. Vermeer ${ }^{1, \dagger}$, Bryant G. Wieking ${ }^{1, \dagger}$, Daniel W. Vermeer ${ }^{1}$ \\ and John H. Lee ${ }^{1,2, *}$
}

1 Cancer Biology Research Center, Sanford Research, Sioux Falls, SD 57104, USA;

E-Mails: ctlucido10@ole.augie.edu (C.T.L.); Paola.Vermeer@SanfordHealth.org (P.D.V.);

Bryant.Wieking@ coyotes.usd.edu (B.G.W.); Daniel.Vermeer@SanfordHealth.org (D.W.V.)

2 Sanford Health, 2301 East 60th Street North, Sioux Falls, SD 57104, USA

$\dagger$ These authors contributed equally to this work.

* Author to whom correspondence should be addressed; E-Mail: John.Lee@ SanfordHealth.org;

Tel.: +1-605-312-6103; Fax: +1-605-312-6201.

Received: 24 October 2014; in revised form: 12 November 2014 / Accepted: 21 November 2014 / Published: 10 December 2014

\begin{abstract}
Standard-of-care cisplatin and radiation therapy (CRT) provides significant tumor control of human papillomavirus (HPV)-mediated head and neck squamous cell carcinomas (HNSCCs); this effectiveness depends on CRT-mediated activation of the patient's own immune system. However, despite good survival, patients suffer significant morbidity necessitating on-going studies to define novel therapies that alleviate this burden. Given the role of the immune system in tumor clearance, immune modulation may further potentiate the CRT-activated response while potentially decreasing morbidity. CD137, an inducible cell surface receptor found on activated $\mathrm{T}$ cells, is involved in differentiation and survival signaling in $\mathrm{T}$ cells upon binding of its natural partner (CD137L). A number of studies have shown the effectiveness of targeting this immune-stimulatory pathway in regards to tumor clearance. Here, we test its role in HPV+ HNSCC tumor clearance using a previously characterized mouse model. We show that amplification of this stimulatory pathway synergizes with CRT for enhanced tumor clearance. Interestingly, tumor clearance is further potentiated by local tumor cell expression of CD137L.
\end{abstract}

Keywords: CD137; immune; HPV; mouse; squamous 


\section{Introduction}

Human papillomavirus (HPV) infection defines a subclass of head and neck squamous cell carcinomas (HNSCCs). Despite presenting with more advanced disease, HPV positive (+) patients typically respond well to standard-of-care combined modality treatments which contributes to better outcomes as compared with their HPV negative (-) counterparts [1]. In fact, HPV status serves as a prognosticator of patient response to therapy and survival. While the incidence of HPV (-) HNSCC is declining, the prevalence of HPV (+) HNSCC cases is on the rise [2,3] making HPV (+) HNSCC patients a growing population. HNSCC patients suffer from significant morbidity; thus, research efforts aim to define new therapy combinations and/or novel drug targets to alleviate this burden. Using a murine HPV (+) HNSCC model, we have previously shown that HPV (+) tumors are curable in mice with combined cisplatin and radiation therapy (CRT), and that this clearance requires an immune response, mediated by $\mathrm{CD}^{+}$and $\mathrm{CD} 8^{+} \mathrm{T}$ cells $[4,5]$.

Immune surveillance cells identify and target tumor cells for destruction [6,7]. Tumor growth and progression, therefore, may indicate an impaired immune response, or, alternatively, changes in the tumor cells themselves that allow for immune evasion [8,9]. While T cells identify a vast array of tumor antigens and consequently target and destroy tumor cells, some tumors escape through a wide variety of immune evasive mechanisms. Our understanding of how standard therapies provide a window of opportunity for altering tumor-mediated immune evasive strategies has significantly increased in recent years, making it an opportune time to develop novel therapies that further enhance immune mediated therapies [10,11]. In fact, immune checkpoint therapies, which augment the immune response, demonstrate promising results in a number of studies [11-13].

CD137 (also 4-1BB), a member of the tumor necrosis factor receptor superfamily (TNFR), is an inducible cell surface receptor expressed by $\mathrm{T}$ cells following activation. Its natural ligand, CD137L (also 4-1BBL), is present on activated antigen-presenting cells (APCs). Signaling via the CD137:CD137L pathway results in activation, differentiation, and preferential survival of $\mathrm{CD}^{+} \mathrm{T}$ cells $[14,15]$. Amplification of this pathway via either activating monoclonal anti-CD137 antibody treatment or CD137L over-expression promotes T cell-mediated tumor rejection [16-18]. Currently, a fully humanized activating monoclonal anti-CD137 antibody has completed phase I and II trials for its anti-tumor properties in the context of melanoma, renal cell carcinoma, and ovarian cancer [19]. Although this approach is promising, these agents require testing in the context for which they will be used. Some adverse reactions, such as inhibition of humoral immunity and severe autoimmune toxicity, have been noted with systemic administration of immune modifying antibodies [20,21]. Challenges with gene transfer of CD137L have also been noted, as the strategy has proven ineffective in several systems where the activating anti-CD137 antibody produced tumor regression [22].

The relative success of $\mathrm{T}$ cell-mediated tumor rejection via CD137:CD137L pathway activation prompted us to test its effectiveness in the context of HPV (+) HNSCC. Given that the increased sensitivity to CRT evident in HPV (+) HNSCC is mediated by an induced immune response, we hypothesized that further enhancing this immune response would increase tumor rejection. Furthermore, as several studies have shown the efficacy of tumor-localized modification of immune regulatory pathways [23-26], we also questioned whether local tumor expression of CD137L would further enhance clearance. To test this hypothesis, we examined the effects of CD137 activation therapies using 
our previously characterized mouse model of HPV (+) HNSCC, comparing the effects of systemic activation (via activating monoclonal anti-CD137 antibody treatment) versus a tumor-localized activation (via overexpression of CD137L on tumor cells). Here, we show that agonistic anti-CD137 antibody synergizes with cisplatin/radiation therapy to decrease tumor growth in vivo. In addition, over-expression of CD137L on tumor cells increases tumor clearance and survival in mice treated with cisplatin/radiation therapy emphasizing the importance of the tumor microenvironment in response to therapy.

\section{Experimental Section}

\subsection{Cells Lines and Cells Culture Conditions}

Mouse oropharyngeal epithelial cells (MOEs) stably expressing HPV16 E6 and E7 together with activated H-Ras and Luciferase (mEERL), were previously derived via retroviral transduction and maintained as previously described [27,28]. mEERL cells are routinely engrafted into mice and have been used as an acceptable animal model for HPV positive disease [13,29]. mEERL cells stably expressing mouse CD137 ligand (mCD137L) were maintained under the same culture conditions as the parental mEERLs, with the inclusion of the appropriate antibiotics.

\subsection{Sub-Cloning of $m C D 137 L$}

pORF-m41BBL v16 (InvivoGen, San Diego, CA, USA), encoding CD137L, was cloned into pcDNA3.1/Zeo (Invitrogen, Grand Island, NY, USA). Briefly, m41BBL (mCD137L) coding sequence was amplified by PCR using the following primers:

Forward primer: 5'-GCGATAGCGAAGCTTCTGAGATCACCGGTAGGAGG-3'.

Reverse primer: 5'-GCGATAGCGTCTAGACCCTGCTCAGACCCCATA-3'.

Following a pre-incubation at $95{ }^{\circ} \mathrm{C}$ for $5 \mathrm{~min}$, cycling conditions were as follows: $95{ }^{\circ} \mathrm{C}$ for $30 \mathrm{~s}$, $65{ }^{\circ} \mathrm{C}$ for $45 \mathrm{~s}, 72{ }^{\circ} \mathrm{C}$ for $2 \mathrm{~min}$ repeating for 30 cycles. Final annealing was $72{ }^{\circ} \mathrm{C}$ for $5 \mathrm{~min}$, and held at $4{ }^{\circ} \mathrm{C}$. The $\mathrm{mCD} 137 \mathrm{~L}$ insert and pcDNA3.1/Zeo vector were cut with Hind III and Xba I (New England Biolabs, Ipswich, MA, USA) followed by ligation and transformation into DH5 $\alpha$ competent cells (Invitrogen). Multiple colonies were analyzed and positive clones were identified through DNA sequencing (Eurofins MWG Operon, Huntsville, AL, USA).

\subsection{Transfection of $m C D 137 L$ into mEERLs}

mEERL cells were transfected with the pcDNA3.1/Zeo mCD137L expression construct using Lipofectamine 2000 (Life Technologies, Grand Island, NY, USA) according to the manufacturer's recommendations. $24 \mathrm{~h}$ post transfection, cells were placed under antibiotic selection using $500 \mu \mathrm{g} / \mathrm{mL}$ Zeocin (Invitrogen) with untransfected mEERLs serving as a control. Cells growing under selection were ring cloned and multiple clones were tested by western blot and immunofluorescence for mCD137L expression. 


\subsection{Western Blot Analysis}

mEERL mCD137L clones were grown to 70\% confluence, rinsed with $1 \times$ PBS and harvested with lysis buffer (50 mM Tris $\mathrm{HCl}$ pH 7.5; $150 \mathrm{mM} \mathrm{NaCl} ; 5$ mM EDTA; 2 mM Na3VO4; $100 \mathrm{mM} \mathrm{NaF}$; $10 \mathrm{mM}$ NaPPi; $10 \%$ glycerol; $1 \%$ Triton; $1 \times$ Halt Protease Inhibitors; $17.4 \mu \mathrm{g} / \mu \mathrm{L}$ PMSF). Membranes were pelleted by centrifugation $\left(10,000 \mathrm{rpm}\right.$ at $\left.4{ }^{\circ} \mathrm{C}\right)$ and soluble proteins harvested. BCA protein assay was performed according to manufacturer's directions (Pierce, Logan, UT, USA) and equal amounts of total protein were separated by SDS-PAGE, transferred to PVDF-membranes (Immobilon-P, Millipore, Billerica, MA, USA), blocked with 5\% BSA, and incubated with the manufacture-recommended concentrations of the primary antibodies: CD137L ((D-20), sc-11819, Santa Cruz Biotechnology, Santa Cruz, CA, USA), GAPDH (AM4300, Life Technologies, Grand Island, NY, USA). Following washes, PVDF membranes were incubated with the appropriate horse radish peroxidase (HRP) conjugated secondary antibody, followed by incubation with substrate (Luminata, Millipore) and developed with a charge-coupled device (CCD) camera imaging system (UVP).

\subsection{Immunofluorescence Staining and Image Acquisition}

Cells were seeded on collagen coated 8-well chamber slides and grown to $80 \%$ confluence. Cells were fixed with $4 \%$ paraformaldehyde (Electron Microscopy Sciences, Hatfield, PA, USA), permeabilized with $0.2 \%$ TritonX-100 (Thermo Scientific, Waltham, MA, USA), blocked in Superblock (Pierce), and incubated with antibody (1:100). Following washes with phosphate buffered saline (PBS), cells were incubated with Alexa Flour-conjugated secondary antibody (Invitrogen), washed, and coverslips mounted with Vectashield plus DaPi (Vector Labs, Burlingame, CA, USA). Cells were analyzed by confocal microscopy (Olympus FlouView 1000, Center Valley, PA, USA).

\subsection{Mice}

Male C57B1/6 mice (The Jackson Laboratory, Bar Harbor, ME, USA) were maintained at the Sanford Research Laboratory Animal Research Facility (LARF) in accordance with USDA guidelines. Experiments were approved by the Sanford Research IACUC and performed within institutional guidelines. Briefly, using a 23-gauge needle, mEERL parental cells, and those over-expressing mCD137 (OE) or minimally expressing mCD137L (ME) were implanted subcutaneously in the right hind flank of mice ( $n=10$ /group for each experiment). Ten to fourteen days post tumor implantation, mice were anesthetized with $87.5 \mathrm{mg} / \mathrm{kg}$ ketamine and $12.5 \mathrm{mg} / \mathrm{kg}$ xylazine, and the hind limb treated locally with 8 Gy X-ray radiation weekly for 3 weeks (RS2000 irradiator, RadSource Technologies, Suwanee, GA, USA). Cisplatin (CalBiochem, Temecula, CA, USA) was dissolved in bacteriostatic 0.9\% sodium chloride (Hospira, Lake Forest, IL, USA) at $20 \mathrm{mg} / \mathrm{m}^{2}$ and administered intraperitoneally concurrent with radiation therapy. For experiments in which mice were treated with antibody, mice were segregated into groups: those receiving anti-CD137 (300 ug/treatment; BioXCell, West Lebanon, NH, USA) or control IgG (400 $\mu \mathrm{g} /$ treatment; BioXCell). Mice were intraperitoneally injected on days 7, 9, and 12 post-tumor implantation. Mice receiving CRT were treated weekly for three weeks beginning at one week post tumor implantation, a treatment schedule which has historically provided good tumor control [4,13]. Tumor growth was measured using previously established techniques [5]. Animals were euthanized 
when tumor size was $>1.5 \mathrm{~cm}$ in any dimension. Mice were considered tumor free when no measurable tumor was detected for a consecutive period of 2 months. Survival graphs were plotted by standardizing each mouse to an endpoint tumor volume of $2000 \mathrm{~mm}^{3}$. Statistical analysis for the survival graphs was performed using the log-rank test. $p$ value less than 0.05 was considered significant.

\section{Results and Discussion}

\subsection{Activating Monoclonal Anti-CD137 Antibody Treatment Inhibits Tumor Growth in Conjunction with Chemotherapy/Radiation}

We have previously demonstrated that an immune response is required for clearance of HPV (+) cancers during treatment with CRT [4]. To determine the effects of an activating monoclonal anti-CD137 antibody in conjunction with standard therapy (cisplatin/radiation), mice were implanted with tumor and treated with activating anti-CD137 antibody alone or in combination with CRT. Mice treated with antiCD137 antibody alone demonstrated no significant effect on tumor growth (Figure 1B) or survival (Figure 1C) as compared to no treatment/IgG controls (Figure 1A) ( $p=0.097$, Figure 1C). Mice treated with activating anti-CD137 antibody in conjunction with CRT demonstrated slowed tumor growth (Figure 1E) when compared with mice treated with CRT alone (Figure 1D). These findings suggest that the immune-mediated effects on tumor growth of HPV (+) tumors evident with CRT can be further enhanced using an immune stimulatory therapy, such as the activating monoclonal anti-CD137 antibody. However, this effect did not significantly improve survival (Figure 1F).

\subsection{When Given with Cisplatin Alone or Radiation Alone, Anti-CD137 Antibody Synergizes Specifically with Cisplatin Treatment}

To determine whether the activating monoclonal anti-CD137 antibody therapy synergizes with either cisplatin or radiation therapy, mice were implanted with mEERL cells and treated with anti-CD137 in combination with either cisplatin or radiation. While anti-CD137 augments antitumor effects of radiation therapy in murine breast and lung carcinoma models [30], our study found no significant improvement on survival ( $p=0.089$ ) when combined with radiation alone (Figure $2 \mathrm{~A}-\mathrm{C}$ ). The combination of cisplatin and anti-CD137, however, resulted in a significant survival improvement compared to cisplatin alone ( $p=0.001$, Figure 2D-F), suggesting that addition of anti-CD137 antibody treatment to cisplatin therapy enhances the antitumor effects of cisplatin.

\subsection{Over-Expression of CD137L on Tumor Cells Significantly Improves Antitumor Effects of Chemotherapy/Radiation}

To determine whether tumor cells themselves activate the CD137 stimulatory pathway via CD137:CD137L interaction, we analyzed endogenous expression of CD137L on mEERL cells. Immunofluorescence and western blot data showed that mEERL cells lack CD137L expression (Figure 3A,D).

Some studies show that CD137L gene transfer elicits significant antitumor effects [31,32]. Others, however, have shown it to be ineffective in a number of systems where anti-CD137 antibody treatment has produced tumor regression [22]. Therefore, to test the role of tumor cell CD137L expression and its potential induction of the CD137:CD137L axis, murine CD137L was stably expressed in mEERL cells 
and individual clones evaluated for CD137L expression via immunofluorescence and Western blotting analysis (Figure 3B-D). One over-expressing (OE) clone was selected for in vivo analysis (Figure 3B,D). Another clone, growing under selection but with minimal CD137L expression (ME), was selected as an additional control (Figure 3C,D).

Figure 1. Activating anti-CD137 antibody administered in conjunction with standard-of-care treatment further inhibits tumor growth. Tumor growth curves (for individual mice) and group Kaplan-Meyer survival curves are shown for controls and for mice receiving treatment. Tumor growth curves for mice receiving (A) control IgG; (B) anti-CD137 antibody; (D) cisplatin/radiation (CRT) alone or (E) CRT in conjunction with anti-CD137 antibody. Kaplan-Meyer survival curves for (C) control mice and those treated with anti-CD137 antibody or (F) control mice receiving CRT or CRT together with anti-CD137 antibody. Arrows indicate three doses of radiation and cisplatin ( 8 Gy radiation and $20 \mathrm{mg} / \mathrm{kg}$ cisplatin on indicated days); asterisks indicate days of anti-CD137 antibody injections.
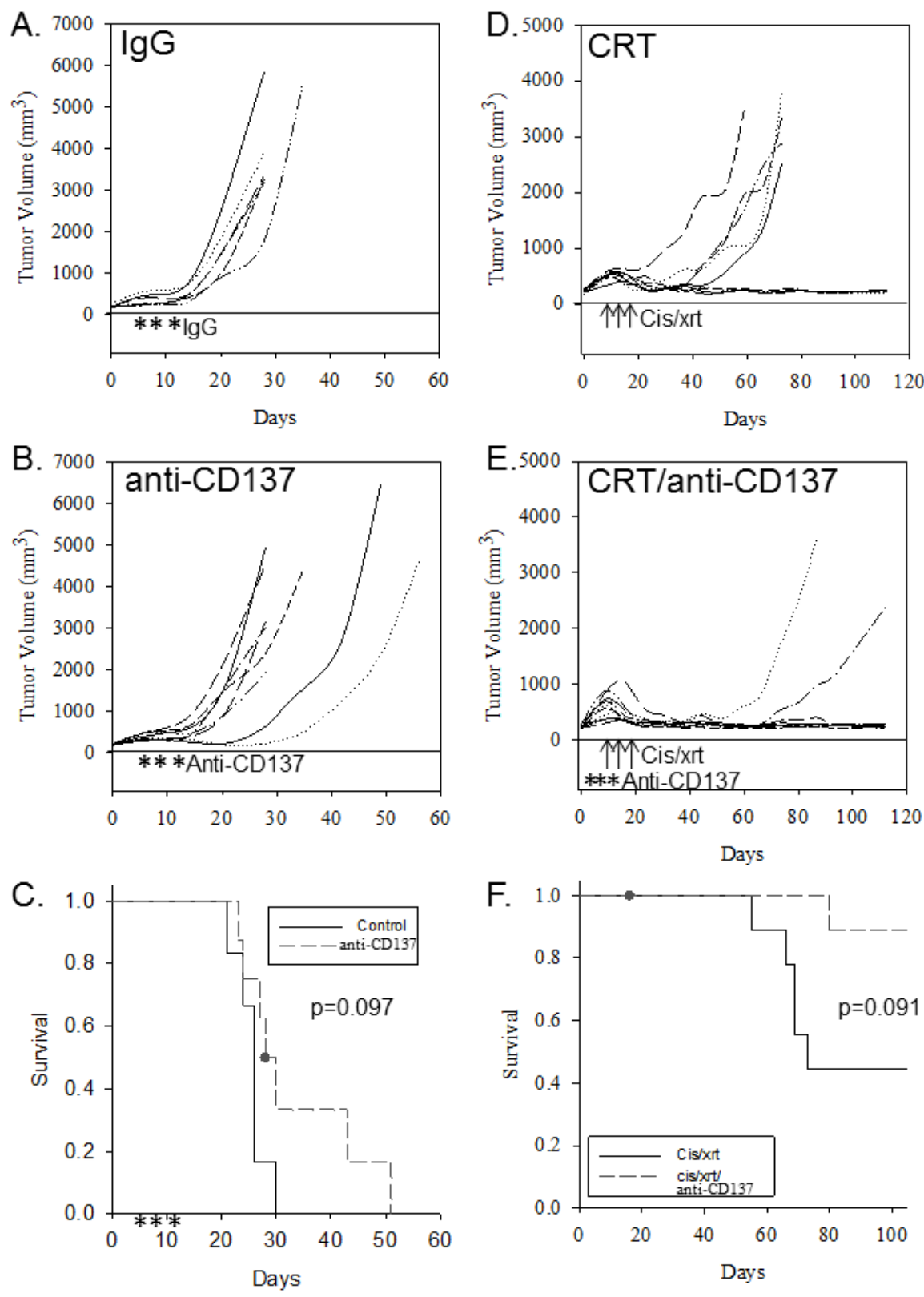
Figure 2. Administration of activating anti-CD137 antibody preferentially enhances the effects of cisplatin treatment. Tumor growth curves (for individual mice) and group Kaplan-Meyer survival curves are shown for mice receiving cisplatin or radiation with IgG, and for mice receiving cisplatin or radiation in conjunction with activating anti-CD137 antibody. (A) Growth curves of mice receiving three doses of radiation ( $8 \mathrm{~Gy}$, on indicated days, arrows) and control IgG (on indicated days, asterisks); (B) Growth curves of mice receiving three doses of radiation (arrows) and anti-CD137 antibody (asterisks); (C) Kaplan-Meyer survival plot comparing mice in A and B; (D) Growth curves of mice receiving three doses of cisplatin $(20 \mathrm{mg} / \mathrm{kg}$, on indicated days, arrows) and control IgG (on indicated days, asterisks); (E) Growth curves of mice receiving cisplatin (arrows) together with anti-CD137 antibody (asterisks); (F) Kaplan-Meyer survival plots of groups in D and E.
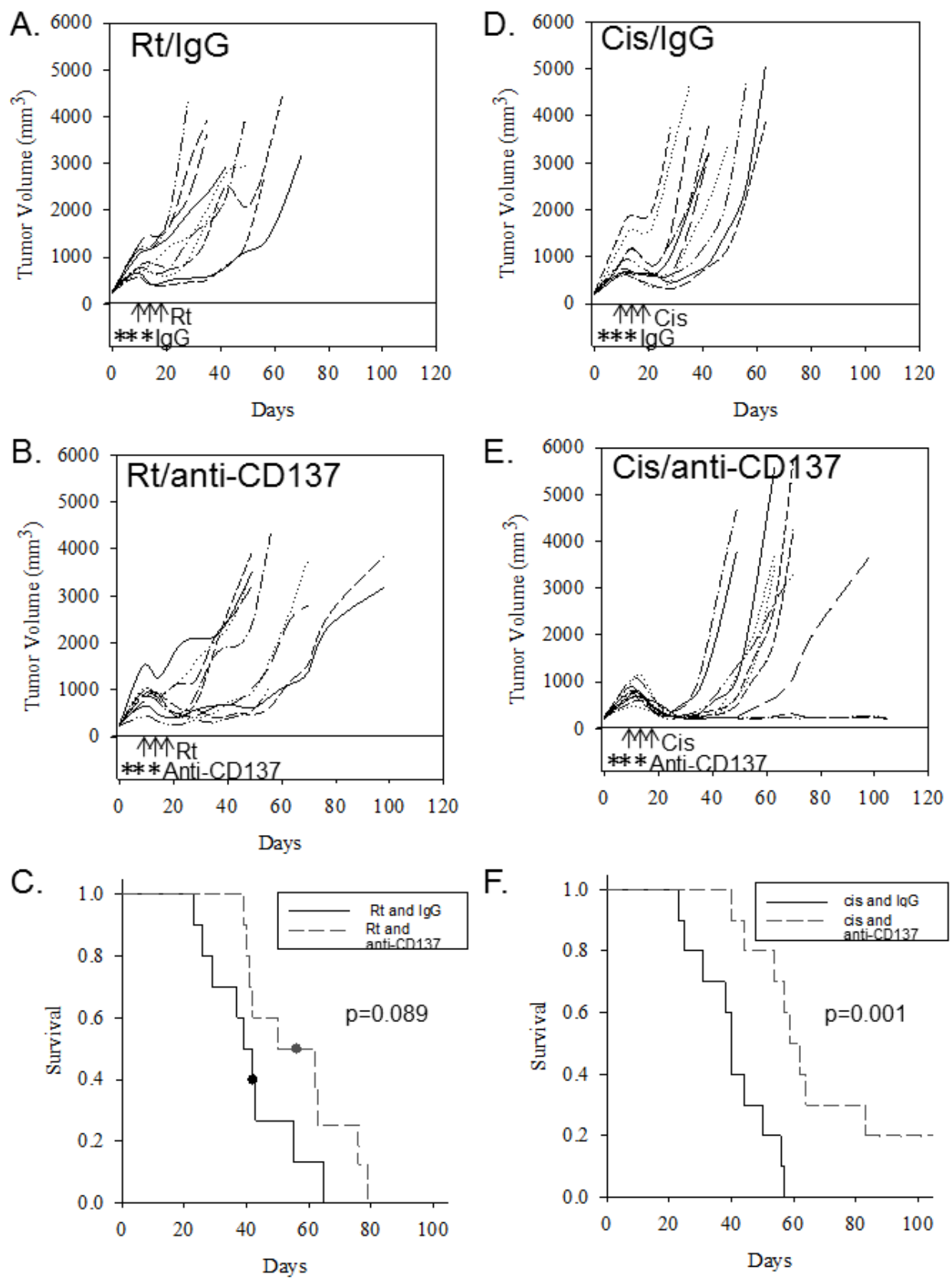
Figure 3. CD137L expression in parental mEERL cells and selected clones. En Face confocal images of mEERL cells and CD137L clones stained for CD137L (green) expression. Immunofluorescent analysis of CD137L expression in (A) parental mEERL cells; (B) a mEERL clone over-expressing CD137L; and (C) a mEERL clone minimally expressing CD137L. Nuclei are counterstained with DaPi (blue); (D) Western blot analysis of CD137L expression. Two additional clones are shown but were not used in these studies.
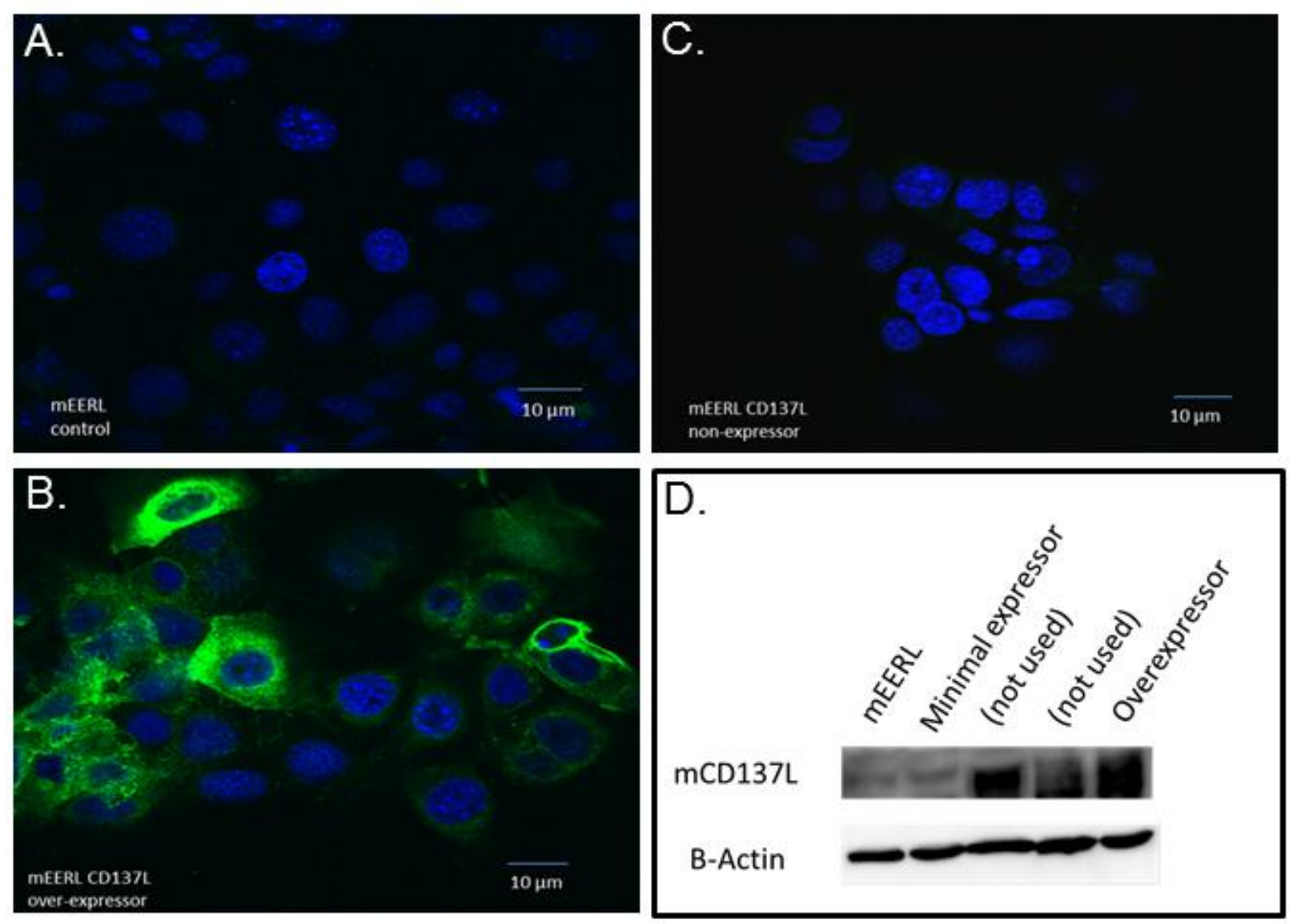

To investigate the effects of tumor CD137L expression on standard-of-care treatment, we implanted these mEERL CD137L clones into mice. We found no statistically significant effect of CD137L expression on tumor growth or survival when mice were not treated with CRT (data not shown). However, treatment with CRT demonstrated a statistically significant improvement $(p<0.001)$ of survival (Figure 4D) and dramatic inhibition of tumor growth in mice with CD137L overexpressing tumors (Figure 4B). Importantly, all mice in this group cleared tumor (Figure 4B). Mice implanted with the CD137 minimally expressing clone responded similarly to CRT as the parental (mEERL) cells (Figure 4C). These findings demonstrate that tumor-localized expression of CD137L together with CRT may not only significantly inhibit tumor growth, but also improve long-term survival. 
Figure 4. Over-expression of CD137L on tumor cells enhances anti-tumor effects of CRT. Tumor growth curves (for individual mice) and group Kaplan-Meier survival curves are shown for mice implanted with (A) parental mEERLs, (B) mEERLs over-expressing CD137L (OE), or (C) mEERLs minimally expressing CD137L (ME). Mice received three doses of cisplatin (20 mg/kg) and radiation (8 Gy) on indicated days (arrows), (D) Kaplan-Meier survival curves for mice in $\mathrm{A}, \mathrm{B}$ and $\mathrm{C}$.
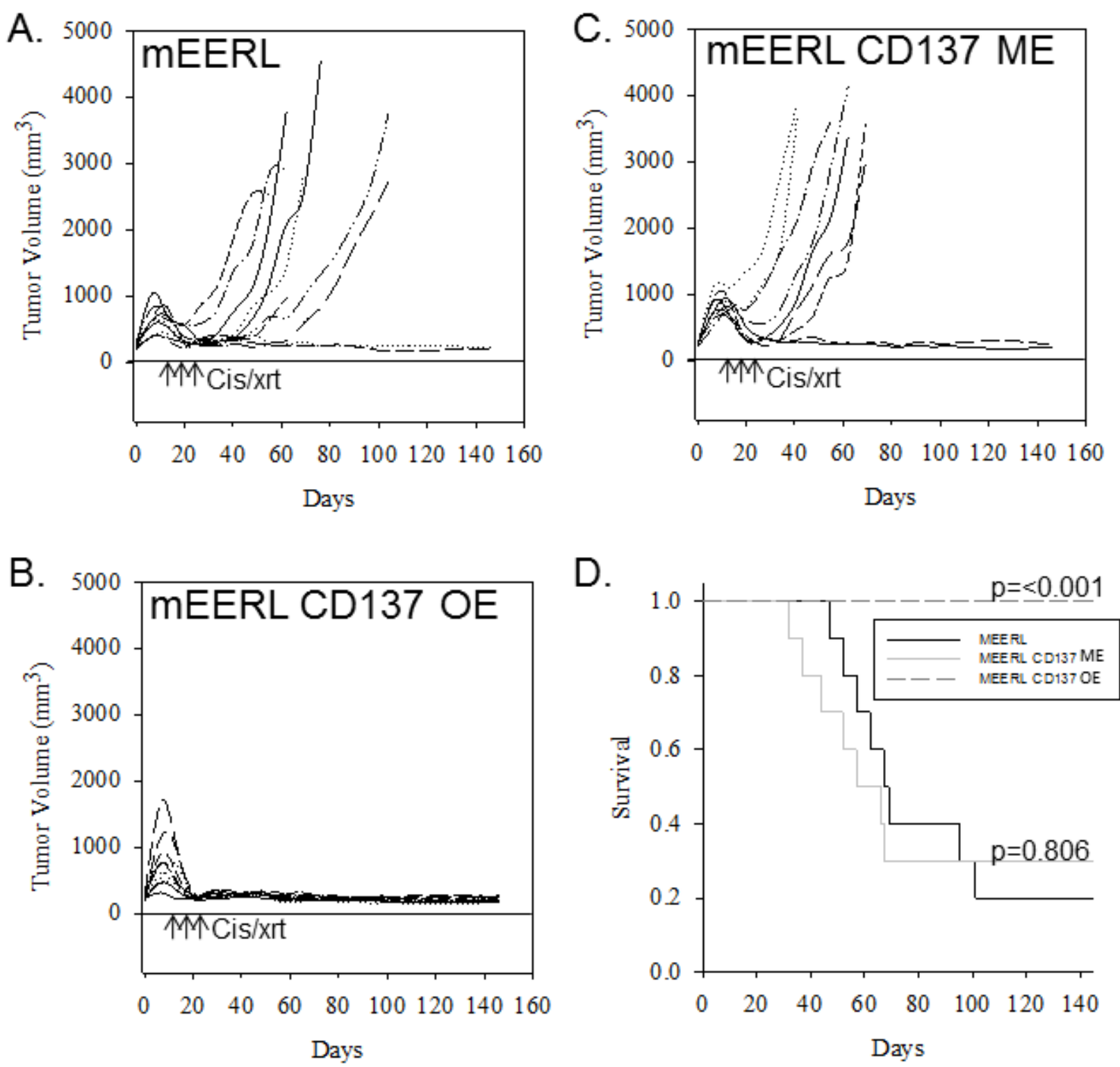

\section{Discussion}

The antitumor effects of immune-modulating therapies have shown promise for various cancers. Therefore, we questioned whether activation of the CD137:CD137L co-stimulatory pathway would enhance immune clearance of HPV (+) cancers either as a single agent, in combination with CRT, and when expressed locally by the tumor cells themselves (i.e., not administered systemically). In this study, we show that agonistic CD137 antibody treatment inhibits tumor growth when given in conjunction with standard CRT treatment in a murine model of HPV+ HNSCC. We demonstrate that this effect is likely mediated by synergy between cisplatin (rather than radiation) and the anti-CD137 antibody. 
Multiple co-stimulatory signals affect immune recognition and activation. Thus, our finding that neither activating monoclonal anti-CD137 antibody treatment nor over-expression of CD137L on tumor cells alone was sufficient to affect tumor clearance is not surprising. In fact, modulating other costimulatory pathways has led to significant clinical success in other pre-clinical models [33] as well as in humans [34]. However, our observation that these strategies improve anti-tumor activity in conjunction with standard CRT suggests a possible route to further augment the immune response activated in HPV (+) HNSCC during CRT. Previous studies have demonstrated the anti-tumor effects of anti-CD137 antibody treatment in combination with radiation [30], however, until now, none have demonstrated its effects in conjunction with cisplatin and radiation in the context of HPV (+) HNSCC. Studies have increasingly recognized the role of HPV in causing cervical, anal, and oropharyngeal tumors. Cisplatin and radiation therapy is commonly used in the treatment of many of these cancers. This study shows the promise of CD137:CD137L signal amplification in stimulating the immune response activated during standard treatment, which is required for HPV (+) tumor clearance. Our findings suggest that further investigation of immune modulatory therapies, in conjunction with standard treatment, could prove efficacious in the context of HPV (+) cancers. These data suggest that strategies of tumor-directed CD137:CD137L amplification should be investigated further as a means to enhance tumor directed immune activity.

\section{Conclusions}

We demonstrate that amplification of the CD137:CD137L pathway synergizes with CRT enhancing tumor clearance in HPV+ HNSCC. Tumor clearance is further potentiated by local tumor cell expression of CD137L. These finding suggest that immune modulatory therapies together with standard treatment can significantly improve outcomes for HPV + cancers and should be further investigated.

\section{Acknowledgments}

The authors thank Catherine Christopherson for excellent administrative assistance and Steven Powell (Sanford Health) for critical reading of the manuscript. This work was supported by R01 DE018386 National Institutes of Health/National Institute of Dental and Craniofacial ResearchNIDCR (to J.H. Lee, B.G. Wieking, D.W. Vermeer and C.T. Lucido) and 8P20GM103548 Center of Biomedical Research Excellence National Institutes of Health/Research Centers in Minority Institutions and Institutional Development Award (to P.D. Vermeer, and B.G. Wieking).

\section{Author Contributions}

Daniel W. Vermeer and John H. Lee conceived and designed experiments; Christopher T. Lucido, Daniel W. Vermeer and Bryant G. Wieking performed the experiments; Paola D. Vermeer and Christopher T. Lucido wrote the manuscript; Daniel W. Vermeer, John H. Lee and Paola D. Vermeer analyzed the data.

\section{Conflicts of Interest}

The authors declare no conflict of interest. 


\section{References}

1. Prince, A.; Aguirre-Ghizo, J.; Genden, E.; Posner, M.; Sikora, A. Head and neck squamous cell carcinoma: New translational therapies. Mt. Sinai J. Med. 2010, 77, 684-699.

2. Shiboski, C.H.; Schmidt, B.L.; Jordan, R.C. Tongue and tonsil carcinoma: Increasing trends in the U.S. population ages 20-44 years. Cancer 2005, 103, 1843-1849.

3. Hammarstedt, L.; Dahlstrand, H.; Lindquist, D.; Onelov, L.; Ryott, M.; Luo, J.; Dalianis, T.; Ye, W.; Munck-Wikland, E. The incidence of tonsillar cancer in Sweden is increasing. Acta Otolaryngol. 2007, 127, 988-992.

4. Spanos, W.C.; Nowicki, P.; Lee, D.W.; Hoover, A.; Hostager, B.; Gupta, A.; Anderson, M.E.; Lee, J.H. Immune response during therapy with cisplatin or radiation for human papillomavirus-related head and neck cancer. Arch. Otolaryngol. Head Neck Surg. 2009, 135, 1137-1146.

5. Williams, R.; Lee, D.W.; Elzey, B.D.; Anderson, M.E.; Hostager, B.S.; Lee, J.H. Preclinical models of HPV+ and HPV- HNSCC in mice: An immune clearance of HPV+ HNSCC. Head Neck 2009, 31, 911-918.

6. Vesely, M.D.; Kershaw, M.H.; Schreiber, R.D.; Smyth, M.J. Natural innate and adaptive immunity to cancer. Annu. Rev. Immunol. 2011, 29, 235-271.

7. Schreiber, R.D.; Old, L.J.; Smyth, M.J. Cancer immunoediting: Integrating immunity's roles in cancer suppression and promotion. Science 2011, 331, 1565-1570.

8. Yaguchi, T.; Sumimoto, H.; Kudo-Saito, C.; Tsukamoto, N.; Ueda, R.; Iwata-Kajihara, T.; Nishio, H.; Kawamura, N.; Kawakami, Y. The mechanisms of cancer immunoescape and development of overcoming strategies. Int. J. Hematol. 2011, 93, 294-300.

9. Seliger, B. Strategies of tumor immune evasion. BioDrugs 2005, 19, 347-354.

10. Shiao, S.L.; Coussens, L.M. The tumor-immune microenvironment and response to radiation therapy. J. Mammary Gland Biol. Neoplas. 2010, 15, 411-421.

11. Curran, M.A.; Kim, M.; Montalvo, W.; Al-Shamkhani, A.; Allison, J.P. Combination CTLA-4 blockade and 4-1BB activation enhances tumor rejection by increasing T-cell infiltration, proliferation, and cytokine production. PLoS One 2011, 6, e19499.

12. Adler, A.J.; Vella, A.T. Betting on improved cancer immunotherapy by doubling down on CD134 and CD137 co-stimulation. Oncoimmunology 2013, 2, e22837.

13. Vermeer, D.W.; Spanos, W.C.; Vermeer, P.D.; Bruns, A.M.; Lee, K.M.; Lee, J.H. Radiation-induced loss of cell surface CD47 enhances immune-mediated clearance of human papillomavirus-positive cancer. Int. J. Cancer 2013, 133, 120-129.

14. Vinay, D.S.; Kwon, B.S. Immunotherapy of cancer with 4-1BB. Mol. Cancer Ther. 2012, 11, 1062-1070.

15. Vinay, D.S.; Kwon, B.S. Role of 4-1BB in immune responses. Semin. Immunol. 1998, 10, 481-489.

16. Melero, I.; Shuford, W.W.; Newby, S.A.; Aruffo, A.; Ledbetter, J.A.; Hellstrom, K.E.; Mittler, R.S.; Chen, L. Monoclonal antibodies against the 4-1BB T-cell activation molecule eradicate established tumors. Nat. Med. 1997, 3, 682-685.

17. Mogi, S.; Sakurai, J.; Kohsaka, T.; Enomoto, S.; Yagita, H.; Okumura, K.; Azuma, M. Tumour rejection by gene transfer of $4-1 \mathrm{BB}$ ligand into a $\mathrm{CD} 80^{+}$murine squamous cell carcinoma and the requirements of co-stimulatory molecules on tumour and host cells. Immunology 2000, 101, 541-547. 
18. Kim, Y.; Strehl, J.W.; Gorschluter, M.; Ziske, C.; Schmidt-Wolf, I.G. Prevention of lethal tumor growth and generation of long-lasting immunity in vivo using CD137L and interleukin-12 gene transfer. In Vivo 2008, 22, 337-343.

19. Sznol, M.; Hodi, F.S.; Margolin, K.; McDermott, D.F.; Ernstoff, M.S.; Kirkwood, J.M.; Wojtaszek, C.; Feltquate, D.; Logan, T. Phase I study of BMS-663513, a fully human anti-CD137 agonist monoclonal antibody, in patients (pts) with advanced cancer (CA). J. Clin. Oncol. 2008, 26, Abstract 3007.

20. Mittler, R.S.; Bailey, T.S.; Klussman, K.; Trailsmith, M.D.; Hoffmann, M.K. Anti-4-1BB monoclonal antibodies abrogate $\mathrm{T}$ cell-dependent humoral immune responses in vivo through the induction of helper T cell anergy. J. Exp. Med. 1999, 190, 1535-1540.

21. Sun, Y.; Chen, J.H.; Fu, Y. Immunotherapy with agonistic anti-CD137: Two sides of a coin. Cell. Mol. Immunol. 2004, 1, 31-36.

22. Hellstrom, K.E.; Hellstrom, I. Therapeutic vaccination with tumor cells that engage CD137. J. Mol. Med. 2003, 81, 71-86.

23. Fransen, M.F.; van der Sluis, T.C.; Ossendorp, F.; Arens, R.; Melief, C.J. Controlled local delivery of CTLA-4 blocking antibody induces $\mathrm{CD}^{+} \mathrm{T}$-cell-dependent tumor eradication and decreases risk of toxic side effects. Clin. Cancer Res. 2013, 19, 5381-5389.

24. Fransen, M.F.; Sluijter, M.; Morreau, H.; Arens, R.; Melief, C.J. Local activation of CD8 T cells and systemic tumor eradication without toxicity via slow release and local delivery of agonistic CD40 antibody. Clin. Cancer Res. 2011, 17, 2270-2280.

25. Jackaman, C.; Nelson, D.J. Intratumoral interleukin-2/agonist CD40 antibody drives CD4 ${ }^{+}$ independent resolution of treated-tumors and $\mathrm{CD} 4^{+}$-dependent systemic and memory responses. Cancer Immunol. Immunother. 2012, 61, 549-560.

26. Kwong, B.; Gai, S.A.; Elkhader, J.; Wittrup, K.D.; Irvine, D.J. Localized immunotherapy via liposome-anchored Anti-CD137 ${ }^{+}$IL-2 prevents lethal toxicity and elicits local and systemic antitumor immunity. Cancer Res. 2013, 73, 1547-1558.

27. Hoover, A.C.; Strand, G.L.; Nowicki, P.N.; Anderson, M.E.; Vermeer, P.D.; Klingelhutz, A.J.; Bossler, A.D.; Pottala, J.V.; Hendriks, W.J.; Lee, J.H. Impaired PTPN13 phosphatase activity in spontaneous or HPV-induced squamous cell carcinomas potentiates oncogene signaling through the MAP kinase pathway. Oncogene 2009, 28, 3960-3970.

28. Wieking, B.G.; Vermeer, D.W.; Spanos, W.C.; Lee, K.M.; Vermeer, P.; Lee, W.T.; Xu, Y.; Gabitzsch, E.S.; Balcaitis, S.; Balint, J.P., Jr.; et al. A non-oncogenic HPV 16 E6/E7 vaccine enhances treatment of HPV expressing tumors. Cancer Gene Ther. 2012, 19, 667-674.

29. Coppock, J.D.; Wieking, B.G.; Molinolo, A.A.; Gutkind, J.S.; Miskimins, W.K.; Lee, J.H. Improved clearance during treatment of HPV-positive head and neck cancer through mTOR inhibition. Neoplasia 2013, 15, 620-630.

30. Shi, W.; Siemann, D.W. Augmented antitumor effects of radiation therapy by 4-1BB antibody (BMS-469492) treatment. Anticancer Res. 2006, 26, 3445-3453.

31. Yurkovetsky, Z.R.; Shurin, G.V.; Barry, D.A.; Schuh, A.C.; Shurin, M.R.; Robbins, P.D. Comparative analysis of antitumor activity of CD40L, RANKL, and 4-1BBL in vivo following intratumoral administration of viral vectors or transduced dendritic cells. J. Gene Med. 2006, 8, $129-137$. 
32. Ye, J.; Li, L.; Zhang, Y.; Zhang, X.; Ren, D.; Chen, W. Recombinant Salmonella-based 4-1BBL vaccine enhances $\mathrm{T}$ cell immunity and inhibits the development of colorectal cancer in rats: In vivo effects of vaccine containing 4-1BBL. J. Biomed. Sci. 2013, 20, doi:10.1186/1423-0127-20-8.

33. Badoual, C.; Hans, S.; Merillon, N.; van Ryswick, C.; Ravel, P.; Benhamouda, N.; Levionnois, E.; Nizard, M.; Si-Mohamed, A.; Besnier, N.; et al. PD-1-expressing tumor-infiltrating T cells are a favorable prognostic biomarker in HPV-associated head and neck cancer. Cancer Res. 2013, 73, 128-138.

34. Seiwert, T.; Burtness, B.; Weiss, J.; Gluck, I.; Eder, J.; Pai, S. A phase Ib study of MK-3475 in patients with human papillomavirus (HPV)-associated and non-HPV-associated head and neck (H/N) cancer. J. Clin. Oncol. 2014, 32, 132361-132144.

(C) 2014 by the authors; licensee MDPI, Basel, Switzerland. This article is an open access article distributed under the terms and conditions of the Creative Commons Attribution license (http://creativecommons.org/licenses/by/4.0/). 